\title{
First-principles calculations on the adsorption behavior of amino acids on a titanium carbide MXene
}

\author{
José D. Gouveia ${ }^{1 a}$, Gerard Novell-Leruth ${ }^{1 a}$, Pedro M. L. S. Reis ${ }^{1}$, Francesc Viñes, ${ }^{2}$ Francesc Illas, ${ }^{2}$ \\ José R. B. Gomes ${ }^{1 *}$ \\ ${ }^{1}$ CICECO - Aveiro Institute of Materials, Department of Chemistry, University of Aveiro, \\ Campus Universitário de Santiago, Aveiro, Portugal \\ ${ }^{2}$ Departament de Ciència de Materials i Química Física \& Institut de Química Teòrica i \\ Computacional (IQTCUB), Universitat de Barcelona, c/ Martí i Franqués 1, 08028 Barcelona, \\ Spain \\ ${ }^{a}$ Equal contribution. \\ *E-mail: jrgomes@ua.pt
}

\section{Abstract}

Due to their vast range of promising biomedical and electronic applications, there is a growing interest in bio-inorganic lamellar nanomaterials. MXenes are one such class of materials, which stand out by virtue of their demonstrated biocompatibility, pharmacological applicability, energy storage performance, and feasibility as single-molecule sensors. Here, we report on first-principles predictions, based on density-functional theory, of the binding energies and ground-state configurations of six selected amino acids (AAs) adsorbed on O-terminated twodimensional titanium carbide, $\mathrm{Ti}_{2} \mathrm{CO}_{2}$. We find that most AAs (aspartic acid, cysteine, glycine and phenylalanine) prefer to adsorb via their nitrogen atom, which forms a weak bond, with a surface $\mathrm{Ti}$ atom, with bond lengths around $2.35 \AA$. In contrast, histidine and serine tend to adsorb parallel to the MXene surface, with their alpha carbon about $3 \AA$ away from it. In both adsorption configurations, the adsorption energies are of the order of the tenths of $\mathrm{eV}$. In addition, we find a positive, nearly linear correlation between the binding energy of each studied AA and its van der Waals volume, which suggests an adsorption dominated by van der Waals forces. The relationship allowed us to predict the adsorption energies for all the proteinogenic AAs on the same $\mathrm{Ti}_{2} \mathrm{CO}_{2}$ MXene. Our analysis additionally shows that in the parallel adsorption mode there is a negligible transfer of charge density from the AA to the surface but noticeable in the $\mathrm{N}$-bonded adsorption mode. In the latter, the isosurfaces of charge density differences show an accumulation of shared electrons in the region between $\mathrm{N}$ and $\mathrm{Ti}$, confirming the predicted $\mathrm{N}-\mathrm{Ti}$ bond. The moderate adsorption energy values calculated, as well as the preservation of the integrity of both the AAs and the surface upon adsorption, reinforce the capability of $\mathrm{Ti}_{2} \mathrm{CO}_{2}$ as a promising reusable biosensor for amino acids.

Keywords: 2D Materials; Adsorption; Amino Acid; Biosensors; Clean MXenes; Density Functional Theory; Titanium Carbide; . 


\section{Introduction}

The isolation of graphene in 2004 triggered the beginning of an era of extensive study of dozens of families of two-dimensional (2D) materials. ${ }^{1}$ In 2011, a new class of 2D materials, comprising transition-metal nitrides, carbides, or carbonitrides, was reported for the first time. ${ }^{2}$ Since these materials are obtained from MAX phases, with details on their synthesis and properties given in a recent review, ${ }^{3}$ they were named MXenes. Briefly, MXenes are constituted by atomic layers of early transition metal elements $(\mathrm{M})$ intercalated by atomic layers of carbon and/or nitrogen $(X)$, with the general formula $M_{n+1} X_{n} T_{x}(n=1-3)$, where $T_{x}$ represents the surface terminations (usually $\mathrm{O}, \mathrm{OH}, \mathrm{F}$, and $\mathrm{H}$ moieties) attached to the external $\mathrm{M}$ layers. ${ }^{4}$ Among other properties, MXenes are characterized by a high conductivity, high oxidation resistance, hydrophilicity, and easily tunable surface termination..$^{4-6}$ These properties make MXenes appropriate for energy storage applications, such as batteries ${ }^{7}$ and supercapacitors, ${ }^{6}$ sensors,${ }^{8}$ have recently been proposed for carbon capture technologies, ${ }^{9-11}$ and employable as heterogeneous catalysts. ${ }^{12-20}$ The present work focuses on the possible biosensing capabilities of these materials taking the O-terminated titanium carbide $\mathrm{Ti}_{2} \mathrm{C}$, hereafter denoted as $\mathrm{Ti}_{2} \mathrm{CO}_{2}$, as a prototype MXene. Although MXenes without surface terminations have been synthesized, ${ }^{21}$ the oxygen termination seems to be one of the most common ones, in special under oxidative environments. Moreover, unlike its hydroxyl counterpart, the oxygen termination allows the effective development of a $2 \mathrm{D}$ material, as it does not lead to the formation of hydrogen bonds coupling MXene layers to each other. Interestingly, $\mathrm{Ti}_{2} \mathrm{CO}_{2}$ has been shown to be suitable for detecting amino acids (namely arginine) via adsorption, ${ }^{22}$ and its interaction with lysozyme has been experimentally studied. ${ }^{23}$ DNA molecules have also been shown to be able to cross $\mathrm{Ti}_{2} \mathrm{CO}_{2}$ nanopores, and DNA transport studies through MXene membranes suggest the potential of this class of 2D materials for DNA translocation and single-molecule sensing applications. ${ }^{24}$ Moreover, the fact that $\mathrm{Ti}_{2} \mathrm{CO}_{2}$ contains the most biocompatible transition metal, makes it readily versatile for biomedical applications. ${ }^{25}$

Despite the demonstrated potential of $\mathrm{Ti}_{2} \mathrm{CO}_{2}$ as a bio-sensor, ${ }^{26}$ to the best of our knowledge, no proofs of concept, aside from the ones mentioned, have been performed, and very little theoretical work has been done regarding the adsorption of amino acids on MXenes, in general, and on this MXene, in particular. A convenient theoretical framework to address this subject is density functional theory (DFT). In fact, DFT based calculations have been used to approach the binding energy of glycine to $\mathrm{Ti}_{3} \mathrm{C}_{2} \mathrm{O}_{2},{ }^{27}$ and the adsorption behavior of valine, arginine, and aspartic acid on other 2D materials such as graphene and hexagonal boron nitride (BN). ${ }^{28}$ In the latter, it was further shown that dispersion corrections to the energy are essential for an accurate description of the adsorption process.

In the present work we focus on the adsorption of six amino acids (AAs) on $\mathrm{Ti}_{2} \mathrm{CO}_{2}$ as described by means of DFT-based calculations. The chosen amino acids are the aspartic acid (ASP), cysteine (CYS), glycine (GLY), histidine (HIS), phenylalanine (PHE), and serine (SER). These were chosen to cover the whole range of polarity/acidity values of amino acids. In particular, we chose amino acids with an aliphatic side chain (GLY and ALA), one aromatic (PHE), an acidic one (ASP), a basic one (HIS), a hydroxylic (SER), and one sulfur-containing (CYS). In the rest of this paper, we first describe the computational details of our calculations, then the respective results, and finally we provide the study conclusions. 


\section{Computational methods}

The DFT based calculations were carried out using the Vienna ab initio simulation package (VASP). ${ }^{29-32}$ The exchange-correlation functional used was the one prescribed by Perdew, Burke, and Ernzerhof (PBE) within the generalized gradient approximation (GGA), ${ }^{33}$ with dispersion corrections included via the so-called D3 method with Becke-Johnson damping, ${ }^{34,35}$ since dispersion is vital for the correct quantitative sizing of the AA adsorption on surfaces. ${ }^{36-38}$ Dipole corrections in the direction perpendicular to the slab were also taken into account. ${ }^{39}$ The explicit treatment of core electrons in the Kohn-Sham equations was avoided by employing the projector-augmented wave (PAW) method ${ }^{40}$, which allows including their effect on the valence electron density. The Kohn-Sham equations were solved using a plane-wave (PW) basis set, with an energy cutoff of $600 \mathrm{eV}$, until the total energy difference between two consecutive iterations was less than $10^{-7} \mathrm{eV}$. Structural relaxations stopped when the total force acting on every atom became smaller than $0.005 \mathrm{eV} \cdot \AA^{-1}$. The Brillouin zone was sampled using a $\Gamma$-centered grid with a distribution of $2 \times 2 \times 1$ k-points. ${ }^{41}$ The choice of these values resulted from a series of convergence tests, ensuring that relative energies were converged within less than $1 \mathrm{meV} \cdot$ atom $^{-1}$. Spin polarization was not considered, as preliminary tests showed it would leave the results unaltered.

We used a rhombic supercell of the same shape and size for all adsorbed AAs on the $\mathrm{Ti}_{2} \mathrm{CO}_{2}$ MXene (Fig. 1). The bulk supercell contained $p(5 \times 5)$ unit cells of $\mathrm{Ti}_{2} \mathrm{CO}_{2}$, with $D_{3 \mathrm{~d}}$ symmetry. The calculated lattice parameter and slab thickness, $a=3.015 \AA$ and $d=4.46 \AA$, respectively, are in good agreement with the values $a=3.04 \AA$ and $d=4.45 \AA$ obtained previously at the PBE level. ${ }^{42}$ In order to avoid interactions in the direction perpendicular to the plane of the slab due to the periodic boundary conditions, the cell height was fixed at $24 \AA$, accounting for approximately $19.5 \AA$ of vacuum, to be partially filled by the AAs.

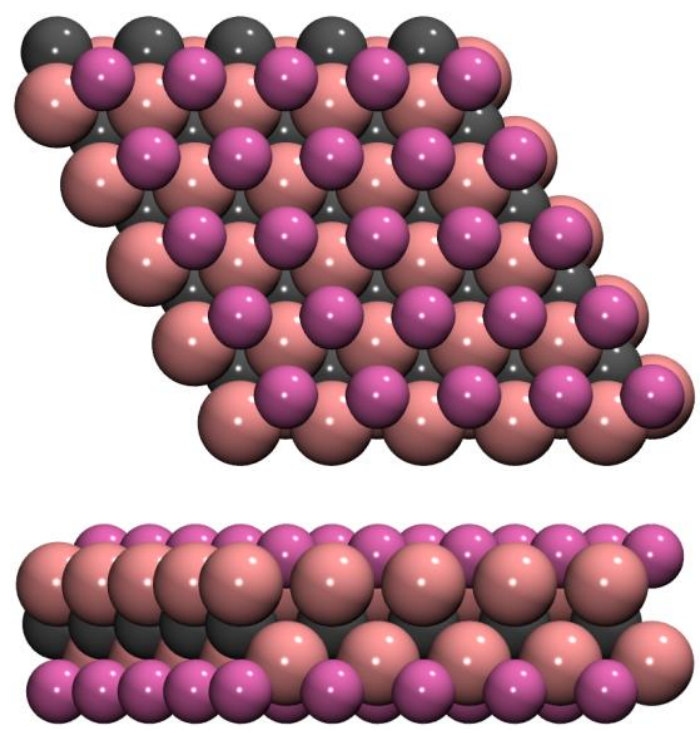

Figure 1 - Top and side views of the $\mathrm{Ti}_{2} \mathrm{CO}_{2}$ supercell. The spheres represent atoms and different colors represent different chemical elements: $\mathrm{C}$ is grey; $\mathrm{O}$ is magenta; and $\mathrm{Ti}$ is pink.

Only L-AAs were sampled, given the achiral character of the $\mathrm{Ti}_{2} \mathrm{CO}_{2}$ surfaces. Several orientations of the molecules relatively to the $\mathrm{Ti}_{2} \mathrm{CO}_{2}$ surface were studied as detailed in Figure S1. In order to speed up the process of finding the most stable orientations on the MXene 
surface, the semiempirical extended tight-binding program package ( $x$ tb, version 6.2 .3$)^{43,44}$ was used for an initial screening. In these calculations, using periodic boundary conditions and default parameters, the $\mathrm{Ti}, \mathrm{C}$ and $\mathrm{O}$ atoms of the MXene were kept frozen while the atomic positions of the AAs were fully optimized. In general, the three most stable structures thus found were then used as initial starting points in the subsequent full-optimization runs with the VASP code. The ground state geometry of each amino acid adsorbed on the surface was then established by comparing the total energy of the local minima configurations of each AA or, equivalently, by picking the one with the most negative adsorption energy, $E_{\text {ads }}$, defined as

$$
E_{\mathrm{ads}}=E_{\mathrm{AA} / \mathrm{MXene}}-\left(E_{\mathrm{MXene}}+E_{\mathrm{AA}}\right)
$$

where $E_{\mathrm{AA} / \mathrm{MXene}}$ is the total energy of the $\mathrm{Ti}_{2} \mathrm{CO}_{2}$ supercell containing the adsorbed $\mathrm{AA}, E_{\mathrm{AA}}$ the energy of the isolated $A A$, calculated in the same unit cell, and $E_{M x e n e}$ the total energy of the pristine $\mathrm{Ti}_{2} \mathrm{CO}_{2}$ slab. As defined, negative values of $E_{\text {ads }}$ refer to stable adsorptions.

The local-minimum character of the ground state configurations was further verified by the normal modes of vibrational analysis of the adsorbed AAs and ensuring that all vibrational frequencies were real. This was done by diagonalization of the corresponding block of the Hessian matrix with elements obtained by finite differences of analytical gradients with displacements of $0.015 \AA$. We investigated the effect of including the degrees of freedom of the surface atoms closest to the adsorbed AAs in frequency calculations, and found that the vibrational modes of the surface are unchanged in the presence of the adsorbed AAs. As we will see, this is to be expected, given the weak adsorbate-adsorbent interaction. For this reason, the surface contribution was neglected in subsequent frequency calculations. The AA frequencies were then used to calculate the partition function and transition-state theory was employed to evaluate the Gibbs free energy for the adsorption process. Representing schematically the process as

$$
A A_{(\mathrm{g})}+* \leftrightarrow A A^{*}
$$

where * stands for a surface active site, the Gibbs free energy of adsorption is given by

$$
G_{\mathrm{ads}}=E_{\mathrm{ads}}-k_{\mathrm{B}} \cdot T \cdot \ln \left(\frac{q_{A A^{*}}}{q_{A A(\mathrm{~g})}}\right)
$$

where $k_{\mathrm{B}}$ is the Boltzmann constant, $T$ the absolute temperature, and $q$ the partition functions of the molecule in gas phase, $A A_{(\mathrm{g})}$, and in its adsorbed form, $A A^{*} .^{45}$ The partition function of the molecule in the gaseous phase ${ }^{46}$ is the product of the vibrational, rotational, translational, and electronic contributions $\left(q=q_{\mathrm{vib}} \cdot q_{\mathrm{rot}} \cdot q_{\text {trans }} \cdot q_{\mathrm{el}}\right)$, while in its adsorbed phase only the vibrational contribution is included, given that the molecular translations and rotations become frustrated because of the adsorption bond. The vibrational partition function can be calculated knowing the frequencies $v_{\mathrm{i}}$ of the normal vibrational modes (Tables S1 and S2 of the Supporting Information),

$$
q_{\mathrm{vib}}=\prod_{i} \frac{\exp \left(-h v_{\mathrm{i}} /\left(2 k_{\mathrm{B}} T\right)\right)}{1-\exp \left(-h v_{\mathrm{i}} /\left(k_{\mathrm{B}} T\right)\right)}
$$

where $h$ is the Planck constant. The rotational and translational contributions ${ }^{45,46}$ to the partition function are given by

$$
q_{\mathrm{rot}} \approx \frac{\sqrt{\pi}}{\sigma} \sqrt{\frac{T^{3}}{\Theta_{r, x} \Theta_{r, y} \Theta_{r, z}}}
$$


and

$$
q_{\text {trans }}=\left(\frac{2 \pi m k_{\mathrm{B}} T}{h^{2}}\right)^{3 / 2} \frac{k_{\mathrm{B}} T}{p}
$$

respectively. Here, $\sigma=1$ is the rotational symmetry number of the AAs, ${ }^{47}$ the $\Theta_{r, i}$ terms are the characteristic rotational temperatures around the $i$ axes $^{48}, m$ the mass of the molecule, and $p$ is the AA partial pressure. In this work and unless noted, the temperature and pressure were fixed at $298.15 \mathrm{~K}$ and $1 \mathrm{bar}$, respectively, and the rotational temperatures of each gaseous AAs were calculated from the diagonalization of their inertia tensors. The procedure for obtaining the molecular partition functions is the same as the one employed in recent studies. ${ }^{49-51}$ The electronic contribution to the partition function, $q_{\mathrm{el}}$ can be approximated to one, given the singlet ground state character of AAs and large energy gap to their excited states.

Lastly, we investigated the possibility of electron transfer between the MXene surface and the adsorbed AAs. To this end, we calculated the Bader charges, ${ }^{52}$ the surface work function, ${ }^{53}$ and the charge density differences between the surface with the adsorbed $A A$ and the infinitely-separated MXene and AA with the same geometry. These concepts and their interpretation are explained in detail in the next section.

\section{Results}

This section is divided in two parts. We first describe in detail our results concerning the mechanism of adsorption of GLY on the titanium carbide MXene, and then present the results for the remaining AAs considered, viz. ALA, ASP, CYS, HIS, PHE, and SER.

\section{Adsorption of glycine}

We studied the GLY molecule (with chemical formula $\mathrm{NH}_{2} \mathrm{CH}_{2} \mathrm{COOH}$ ) in its two most stable conformations, ${ }^{54} \mathrm{I}$ and II, shown in the two top panels of Figure 2. These two conformations differ by the nature of the internal hydrogen bonds, i.e., O-H $\cdots \mathrm{N}$ in Gly-I and $\mathrm{N}-\mathrm{H} \cdots \mathrm{O}$ in Gly-II . According to our calculations, the two molecular conformations are nearly degenerate in gas phase, GLY-I being slightly more stable, by $51 \mathrm{meV}$, a value which is of the order of the error bar of the calculations. Conformation I was therefore taken as reference for adsorption energy calculations. Several adsorption configurations were compared, as shown in Figure S1 of the Supporting Information ( $\mathrm{SI}$ ). Here, we focus on the configurations displaying the strongest adsorption strength, shown in Figure 2.

When adsorbing parallel to the surface, GLY sits with its alpha carbon atom at $\sim 3 \AA$ away from the plane defined by the surface $O$ atoms. The adsorption occurs with the amine group of GLY near a surface $\mathrm{O}$ atom, with one of its $\mathrm{H}$ atoms pointing towards that $\mathrm{O}$ atom. One of the $\mathrm{H}$ atoms in the alpha carbon is oriented towards the surface while the other is pointing in the direction of the vacuum region. The carboxylic group $(\mathrm{COOH})$ remains parallel to the surface. The adsorption energies for conformations I and II of GLY, both in the mode parallel to the surface, are -0.52 and $-0.42 \mathrm{eV}$, respectively.

The GLY-II conformation can also adsorb on the $\mathrm{Ti}_{2} \mathrm{CO}_{2}$ surface by its nitrogen atom, keeping the plane defined by the alpha carbon with amino and carboxyl group , $\mathrm{H}_{2} \mathrm{~N}-\mathrm{C}_{\alpha^{-}}$ $\mathrm{C}(=\mathrm{O}) \mathrm{OH}$, moiety normal to the surface (Figure 2, bottom panels). This configuration was found to be the most stable for GLY interacting with this MXene, with an adsorption energy of -0.55 eV. Note that the most stable GLY adsorption configuration on the $\mathrm{Ti}_{2} \mathrm{CO}_{2}$ surface is the same 
as the one reported previously on $\mathrm{Ti}_{3} \mathrm{C}_{2} \mathrm{O}_{2}$, with the $\mathrm{GLY}$ adsorbate perpendicular to the surface plane and oriented with its amine moiety towards the surface. ${ }^{27}$ In the case of the most favorable adsorption configuration, the nearest surface Ti atom is pulled $\sim 0.35 \AA$ out of the $\mathrm{Ti}$ plane and towards the amino acid, so that the $\mathrm{N}$-Ti distance becomes $2.35 \AA$, which is the same value found in Ref. ${ }^{27}$. The short distance and the quite large adsorption energy suggest a chemical bond between GLY and the MXene surface, as will be analyzed in detail below. The parallel adsorptions of GLY display positive values of $G_{\text {ads, }}$ around $70 \mathrm{meV}$ at ambient temperature and pressure $(298.15 \mathrm{~K}$ and $1 \mathrm{~atm})$, while the perpendicular adsorption has a $G_{\text {ads }}$ of zero.

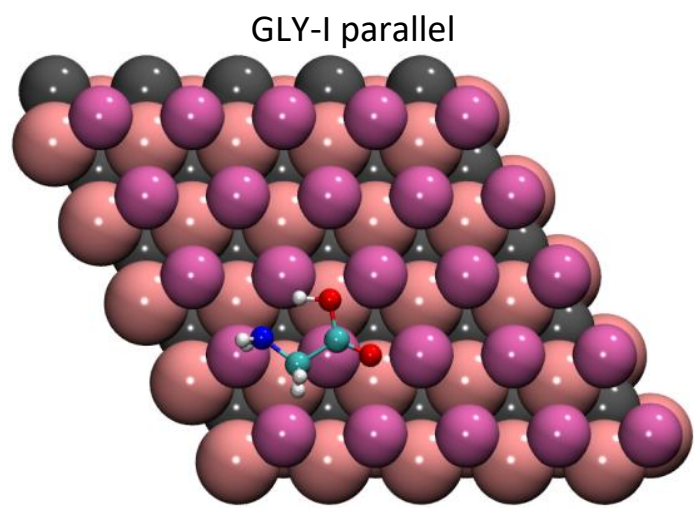

GLY-II perpendicular

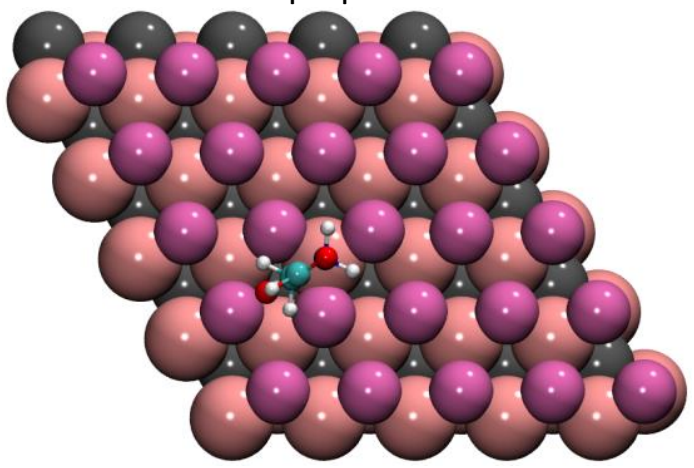

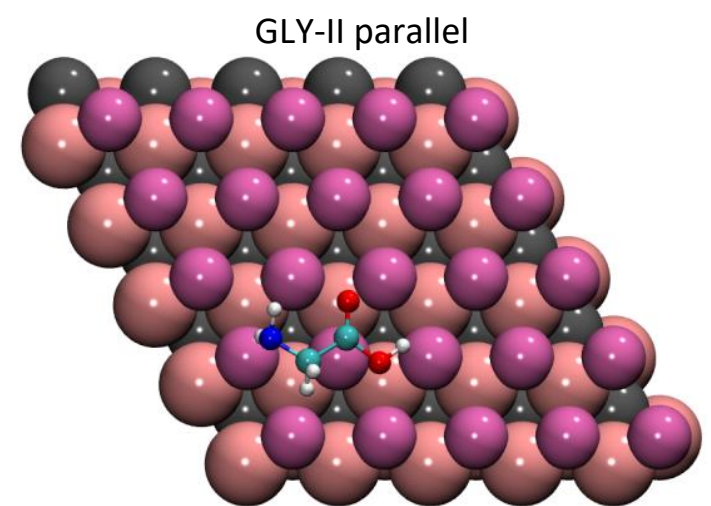

GLY-II perpendicular

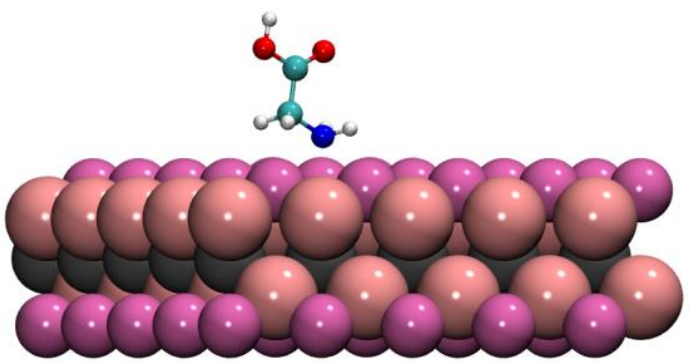

Figure 2 - Several stable adsorbed configurations of GLY on the $\mathrm{Ti}_{2} \mathrm{CO}_{2} \mathrm{MXene}$ surface. The top panels depict parallel adsorption of GLY in its two most stable conformations (I and II). ${ }^{54}$ The bottom panels show the top (left) and side (right) views of the perpendicular adsorption configuration of GLY-II. The color code for the MXene atoms is the same as in Fig. 1. Color code for the smaller spheres (Gly atoms): $\mathrm{H}$ is white; $\mathrm{C}$ is cyan; $\mathrm{N}$ is blue, and $\mathrm{O}$ is red.

For the sake of comparison with the results of Ref. ${ }^{27}$, we briefly compare the adsorption of $\mathrm{GLY}$ on the $\mathrm{Ti}_{3} \mathrm{C}_{2} \mathrm{O}_{2} \mathrm{MXene}$ surface. We found that, as on $\mathrm{Ti}_{2} \mathrm{CO}_{2}$, the strongest adsorption is found when the molecule is perpendicular to the surface and bound by its $\mathrm{N}$ atom to a Ti atom of the surface (see Figure S2), with an adsorption energy of $-0.50 \mathrm{eV}$ using the PBE-D3 functional, or $-0.57 \mathrm{eV}$ with the PBE-optB86b functional. The difference between these values is within the error bar of the calculations, and therefore they can be considered identical to each other and to the calculated adsorption energy on $\mathrm{Ti}_{2} \mathrm{CO}_{2}$, suggesting that the thickness of the MXene surface has no significant effect on the mechanism or strength of $A A$ 
adsorption, as also seen in other studies, e.g. on the $\mathrm{CO}_{2}$ capture with MXenes with different width. ${ }^{55}$

The calculated vibrational frequencies of GLY in gaseous phase or adsorbed on the $\mathrm{Ti}_{2} \mathrm{CO}_{2}$ are shown in Table S1. The frequencies of gaseous GLY can be compared to those in Ref. ${ }^{56}$, where infrared (IR) spectra were recorded for several GLY conformers, including GLY-I, our gas-phase reference. For the gaseous phase GLY-I, our calculated frequencies are systematically lower than the measured ones, although by $16 \mathrm{~cm}^{-1}$ on average only. In addition, we find two frequencies, associated to $\mathrm{CCH}$ bending modes, in the $1300 \mathrm{~cm}^{-1}$ region, which are absent from the list of measured frequencies, an effect observed in Ref. ${ }^{56}$ as well. The mode related to $\mathrm{CN}$ stretch at $1050 \mathrm{~cm}^{-1}$ calculated by us is also absent from the experimental spectrum and has correspondence with the $1059 \mathrm{~cm}^{-1}$ frequency calculated in Ref. ${ }^{56}$.

In contrast to the above results for the gas phase GLY conformers, the calculated normal mode frequencies of the adsorbed GLY-II differ from experiment by up to $70 \mathrm{~cm}^{-1}$. The calculated frequencies (and respective IR intensities) for adsorbed GLY can be compared with the ones in the IR spectrum obtained experimentally for $\mathrm{GLY}$ on $\mathrm{Ti}_{3} \mathrm{C}_{2} \mathrm{~T}_{\mathrm{x} .}{ }^{27}$ Table $\mathrm{S} 3$ summarizes these comparisons and Figure $\mathrm{S} 4$ shows the IR spectrum of GLY-II adsorbed perpendicularly on $\mathrm{Ti}_{2} \mathrm{CO}_{2}$, calculated using the method in Ref. ${ }^{57}$. This plot shows that the modes predicted to have the highest IR intensities are the ones at 1755, 1361, 1276, 1136, 1091, and $1028 \mathrm{~cm}^{-1}$. The experimental band observed at $1640 \mathrm{~cm}^{-1}$ for GLY on $\mathrm{Ti}_{3} \mathrm{C}_{2} \mathrm{~T}_{\mathrm{x}}$ which was assigned to $\mathrm{NH}_{2}$ bending, is blue-shifted by around $20 \mathrm{~cm}^{-1}$ upon adsorption. The calculated value is $1599 \mathrm{~cm}^{-1}$ but its intensity is very low and, therefore, it is absent from the calculated spectrum. There are plenty of explanations for this discrepancy, related to the cleanness of the surface, the presence of a solvent, and the adsorption mechanism. Firstly, here we assume the $\mathrm{Ti}_{2} \mathrm{C}$ surface is terminated by a single layer of $\mathrm{O}$, while the experiments in Ref. ${ }^{27}$ were conducted using $\mathrm{Ti}_{3} \mathrm{C}_{2} \mathrm{~T}_{\mathrm{x}}$, where the $\mathrm{T}_{\mathrm{x}}$ termination is mostly unknown and may include $-\mathrm{O},-\mathrm{OH}$, or $-\mathrm{F}$. Secondly, the calculations were performed assuming ultrahigh vacuum, which differs significantly from the experimental conditions in Ref. ${ }^{27}$. Thirdly, the most favored adsorption configuration of GLY might not be as simple as a single adsorbed molecule, isolated from all other GLY molecules.

\section{Adsorption of other amino acids}

After the GLY test case, we studied the adsorption mechanism of ASP, CYS, HIS, PHE, and SER on $\mathrm{Ti}_{2} \mathrm{CO}_{2}$. The most stable adsorption configuration of each AA was found after energetically comparing orientations analogous to the ones analyzed for GLY, as shown in Figures 2 and S1. As in the case of GLY, the two most stable adsorption configurations of each AA are the parallel one and the one which allows a chemical bond between the nitrogen atom of the AA and the nearest surface Ti atom. Figure 3 shows the optimized geometries of each adsorbed AA, both parallel and $\mathrm{N}$-bonded to the surface. 


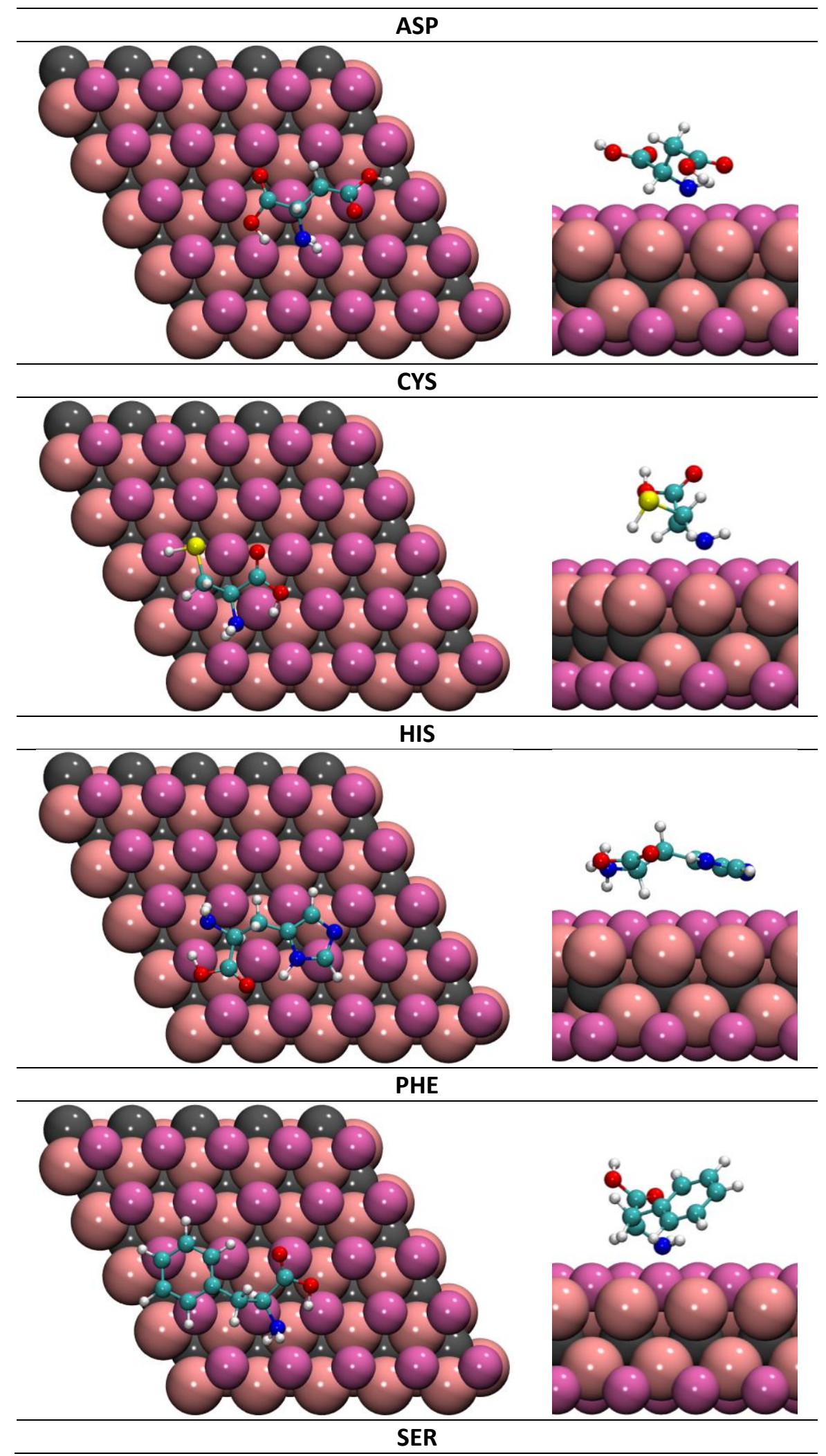




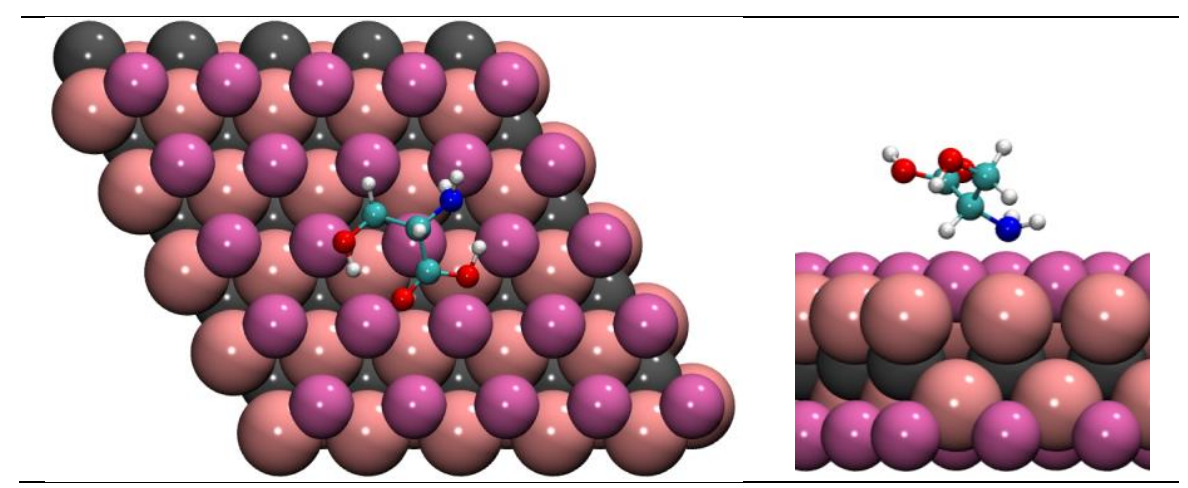

Figure 3 - Ground state adsorbed configurations of the six studied AAs. A top view of the parallel adsorption is shown on the left, and a side view of the $\mathrm{N}$-bonded adsorption on the right. The color code is the same as that of Figure 2 and $S$ is yellow.

The parallel adsorptions occur with the nearest atom (a hydrogen in all cases) about 2 $\AA$ away from substrate, which remains virtually unaffected by the presence of the AA. The amino groups of ASP and SER lie near an $\mathrm{O}$ surface atom with one of its $\mathrm{H}$ atoms pointing towards that $\mathrm{O}$ atom while the alpha $\mathrm{C}$ stays near another surface $\mathrm{O}$ atom. This contrasts with CYS and HIS where the amine group is on top of a Ti atom and the alpha $\mathrm{C}$ lies above a carbon hollow site of the surface. In the case of PHE, the $\mathrm{N}$ and the alpha $\mathrm{C}$ are both near Ti atoms of the surface and the $\mathrm{H}$ of the alpha $\mathrm{C}$ is the atom closest to the surface. Interestingly, the $\mathrm{H}$ atom in the alpha carbon of CYS, HIS, and PHE is pointing towards the surface while in ASP and SER the same atom is pointing away from the surface. However, the energy differences between the configurations with the $\mathrm{H}$ atom in the alpha carbon pointing towards or away the surface are below $0.1 \mathrm{eV}$. Regarding the $\mathrm{N}$-bonded adsorption, from inspection of the righthand side panels of Figure 3, one readily notices that, like in the case of GLY adsorption, the nearest $\mathrm{Ti}$ atom is drawn towards the nitrogen atom of all the AAs. Nonetheless, unlike GLY, the larger AAs do not remain truly perpendicular to the surface, but instead attempt to return to their parallel orientation, while keeping the $\mathrm{N}$ and $\mathrm{Ti}$ atoms close to each other. The possibility of an $\mathrm{N}-\mathrm{Ti}$ bond is analyzed in detail below. As we will see, this configuration is advantageous on some AAs, while others prefer the parallel alignment. Table 1 contains relevant calculated properties of the adsorbed AAs. All adsorption energies $\left(E_{\text {ads }}\right)$ are negative, regardless of the relative orientation of the $A A$ and the surface, indicating favorable adsorption in both situations. Interestingly, some AAs prefer parallel adsorption, while others prefer the $\mathrm{N}$-bonded one; furthermore, on some AAs, the relative stability is reversed at ambient temperature and pressure. 
Table 1 - Relevant physicochemical quantities for the adsorption of the studied AAs on $\mathrm{Ti}_{2} \mathrm{CO}_{2}$. The data include the adsorption energy $\left(E_{\text {ads }}\right)$, Gibbs free energy of adsorption ( $\left.G_{\text {ads }}\right)$ of the $\mathrm{AA}$ at $298 \mathrm{~K}$ and $1 \mathrm{bar}$, and of the Bader charge $(\Delta Q)$ of the surface unit cell when in the presence of each adsorbed amino acid. For each AA, the lowest $E_{\text {ads }}$ and the lowest $G_{\text {ads }}$ among the parallel and $\mathrm{N}$-bonded adsorptions are highlighted in bold.

\begin{tabular}{|c|c|c|c|c|c|c|c|c|c|c|}
\hline \multirow[b]{2}{*}{ AA } & \multirow[b]{2}{*}{$\begin{array}{c}V \\
\left(\AA^{3}\right)^{a}\end{array}$} & \multicolumn{4}{|c|}{ Parallel adsorption } & \multicolumn{5}{|c|}{$\mathrm{N}$-bonded adsorption } \\
\hline & & $\begin{array}{l}d_{\alpha-O} \\
(\AA)^{b}\end{array}$ & $\begin{array}{l}E_{\text {ads }} \\
(\mathrm{eV})\end{array}$ & $\begin{array}{c}G_{\mathrm{ads}} \\
(\mathrm{eV})\end{array}$ & $\begin{array}{l}\Delta Q \\
(e)\end{array}$ & $\begin{array}{c}d_{\mathrm{N}-\mathrm{Ti}} \\
(\AA)^{\mathrm{c}}\end{array}$ & $\begin{array}{c}\mathrm{N}-\mathrm{Ti} \\
\text { bond }^{\mathrm{d}}\end{array}$ & $\begin{array}{l}E_{\text {ads }} \\
(\mathrm{eV})\end{array}$ & $\begin{array}{l}G_{\text {ads }} \\
(\mathrm{eV})\end{array}$ & $\begin{array}{l}\Delta Q \\
(e)\end{array}$ \\
\hline ASP & 91 & 3.42 & -0.79 & -0.23 & 0.00 & 2.33 & 0.169 & -0.88 & -0.18 & 0.19 \\
\hline CYS & 86 & 2.79 & -0.67 & -0.18 & 0.09 & 2.42 & 0.137 & -0.69 & -0.04 & 0.18 \\
\hline GLY & 48 & 3.08 & -0.53 & 0.00 & 0.01 & 2.35 & 0.161 & -0.55 & 0.07 & 0.19 \\
\hline HIS & 118 & 2.85 & -0.91 & -0.35 & 0.16 & 2.33 & 0.168 & -0.76 & -0.14 & 0.23 \\
\hline PHE & 135 & 3.00 & -0.94 & -0.43 & 0.07 & 2.35 & 0.161 & -0.98 & -0.34 & 0.21 \\
\hline SER & 73 & 3.46 & -0.60 & -0.04 & 0.01 & 2.33 & 0.169 & -0.47 & 0.14 & 0.20 \\
\hline ALA & 67 & 3.53 & -0.55 & -0.02 & 0.01 & 2.33 & 0.170 & -0.65 & -0.03 & 0.21 \\
\hline
\end{tabular}

${ }^{a}$ The van der Waals volumes $(V)$ of the AAs were taken from Ref. ${ }^{58}$.

${ }^{b}$ Distance between the alpha carbon of the AA and the nearest oxygen surface layer.

${ }^{c}$ Distance between the $\mathrm{N}$ atom of the $\mathrm{AA}$ and its nearest surface Ti atom $\left(d_{\mathrm{N}-\mathrm{Ti}}\right)$.

${ }^{d}$ Bond order between the $\mathrm{N}$ atom of the $\mathrm{AA}$ and its nearest surface Ti atom, calculated using the method in Ref. ${ }^{59}$.

For the parallel adsorption, the distances between the alpha carbon of each AA and the carbon layer of $\mathrm{Ti}_{2} \mathrm{CO}_{2}\left(d_{\alpha-0}\right)$ were calculated in the direction perpendicular to the surface. There is clearly no correlation between this distance and the adsorption energies, as the AAs with the most similar adsorption energies, ASP and CYS, are the ones with highest and lowest $d_{\alpha-0}$, respectively. The same lack of correlation is observed between the adsorption energies and $\mathrm{N}$-Ti distances $\left(d_{\mathrm{N}-\mathrm{Ti}}\right)$ in the case of $\mathrm{N}$-bonded adsorption. However, the overall results also hint that the largest AAs adsorb more strongly to the surface. As can be seen in Figure S3, the parallel adsorption stability of the AAs increases in the order GLY $<$ SER $<$ CYS $<$ ASP $<$ HIS $<$ PHE, which coincides with the order of molar masses of the AAs. This is to be expected for an interaction dominated by van der Waals ( $v d W$ ) forces, which is favored by a large number of contacts with subtle differences found in the calculated infrared spectra (Figure S6) that seem to be originated by the composition of the AA lateral chain. To verify this assertion, we investigated the correlation between the calculated binding energies of the AAs and their van der Waals volumes $(V) .{ }^{58}$ The linear correlation is evident from Fig. 4, via the represented linear regression line and the respective correlation coefficient of $R^{2}=0.87$. The Gibbs free energies of adsorption ( $\Delta G_{\text {ads }}$ ) also display a good linear correlation with the van der Waals volumes, with $R^{2}=0.96$. This correlation reinforces the claim regarding the dominant role of van der Waals interactions in the adsorption process, as the strength of these interactions increases with the size of the interacting molecules. The adsorption energy of a seventh amino acid (alanine - ALA) was also calculated and included in the plot, with its most stable ( $\mathrm{N}$ bonded) adsorption configuration shown in Figure 5 . We found that alanine fits the trend found for the original six AAs (Figure 4) with a difference between the calculated $E_{\text {ads }}$ value ($0.65 \mathrm{eV}$, Table 1$)$ and the one predicted with the fitted equation (-0.64 eV, Table 2$)$ of only 0.01 $\mathrm{eV}$. The lines represented for $E_{\text {ads }}$ and $G_{\text {ads }}$ have very similar slopes, suggesting that the extra entropy-related term involved in the calculation of $G_{\text {ads }}$ is practically independent of the AA 
and approximately equal to $0.56 \mathrm{eV}$. Using the calculated relationships $E_{\text {ads }}=\mathrm{f}\left(V_{\mathrm{vdw}}\right)$ and $G_{\text {ads }}=f\left(V_{\mathrm{vdw}}\right)$ in Figure 4, we predicted the adsorption energies and Gibbs free energies of adsorption of the remaining proteinogenic amino acids on $\mathrm{Ti}_{2} \mathrm{CO}_{2}$, as shown in Table 2. The adsorption energies are between $-0.53 \mathrm{eV}$ and $-1.15 \mathrm{eV}$, with the largest $\mathrm{AA}$, tryptophan, having the strongest adsorption.

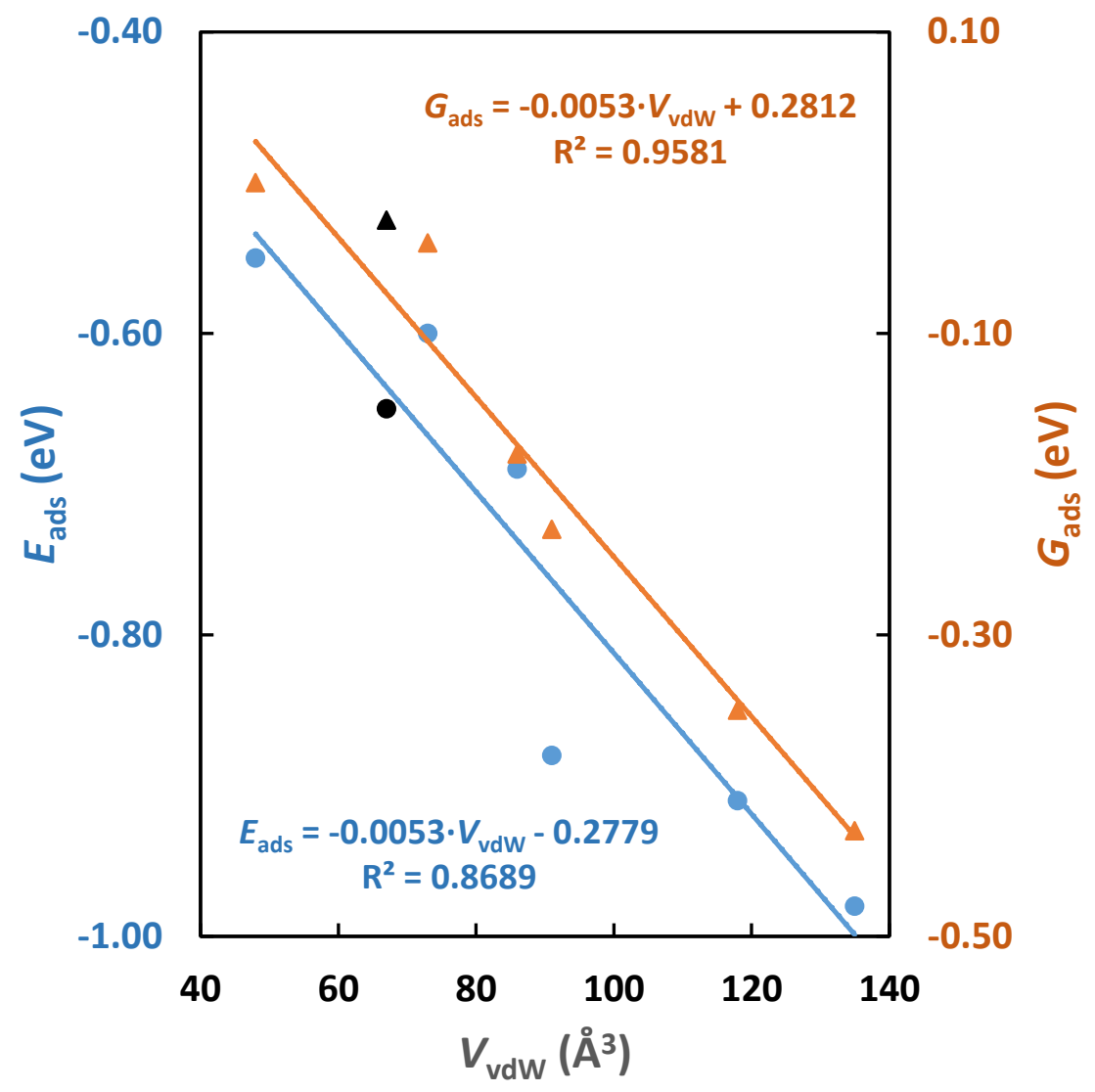

Figure 4 - Plot of the calculated adsorption energies (blue dots) and adsorption Gibbs energies (orange triangles) of the six studied amino acids (blue dots) as a function of their van der Waals volumes and their respective linear regression lines and fitted equations. The black dot/triangle corresponds to the adsorption energy/Gibbs energy of a seventh amino acid, alanine, not included in the fitting (see text for details).

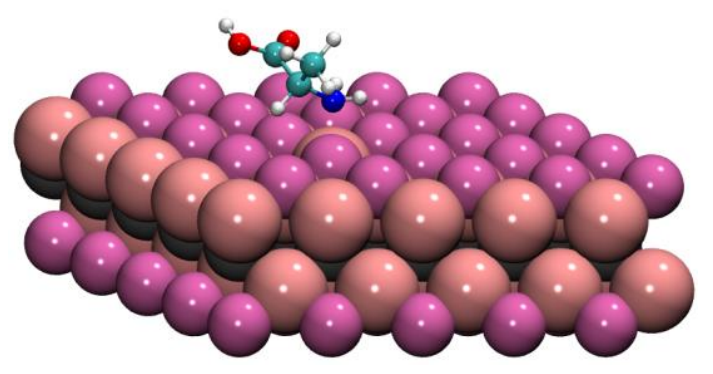


Figure 5 - Ground state configuration of ALA adsorbed on the $\mathrm{Ti}_{2} \mathrm{CO}_{2}$ surface. Color code as in Figure 2.

Table 2 - Calculated adsorption energies $\left(E_{\text {ads }}\right)$ and Gibbs free energies of adsorption $\left(G_{\text {ads }}\right)$ of the twenty amino acids on $\mathrm{Ti}_{2} \mathrm{CO}_{2}$, extrapolated from the relationship $E_{\mathrm{ads}}=-0.0053 \cdot \mathrm{V}_{\mathrm{vdw}}-$ 0.2779 and $G_{\text {ads }}=-0.0053 \cdot V_{\text {vdw }}+0.2812$.

\begin{tabular}{|c|c|c|c|}
\hline AA & $\begin{array}{c}\boldsymbol{V}_{\text {vdW }} \\
\left(\AA^{3}\right)\end{array}$ & $\begin{array}{c}E_{\text {ads }} \\
(\mathbf{e V})\end{array}$ & $\begin{array}{c}\boldsymbol{G}_{\text {ads }} \\
(\mathbf{e V})\end{array}$ \\
\hline ALA & 67 & -0.64 & -0.07 \\
\hline ARG & 148 & -1.07 & -0.50 \\
\hline ASN & 96 & -0.79 & -0.23 \\
\hline ASP & 91 & -0.76 & -0.20 \\
\hline CYS & 86 & -0.74 & -0.17 \\
\hline GLN & 114 & -0.89 & -0.32 \\
\hline GLU & 109 & -0.86 & -0.30 \\
\hline GLY & 48 & -0.53 & 0.03 \\
\hline HIS & 118 & -0.91 & -0.34 \\
\hline ILE & 124 & -0.94 & -0.38 \\
\hline LEU & 124 & -0.94 & -0.38 \\
\hline LYS & 135 & -1.00 & -0.43 \\
\hline MET & 124 & -0.94 & -0.38 \\
\hline PHE & 135 & -1.00 & -0.43 \\
\hline PRO & 90 & -0.76 & -0.20 \\
\hline SER & 73 & -0.66 & -0.11 \\
\hline THR & 93 & -0.77 & -0.21 \\
\hline TRP & 163 & -1.15 & -0.58 \\
\hline TYR & 141 & -1.03 & -0.47 \\
\hline VAL & 105 & -0.84 & -0.27 \\
\hline
\end{tabular}

The adsorptions of GLY and PHE on the reduced $\mathrm{Ti}_{2} \mathrm{C}$ surface, i.e., that obtained from the $\mathrm{Ti}_{2} \mathrm{CO}_{2} \mathrm{MXene}$ upon removal of the terminating oxygen atoms, were also analyzed in this work. As expected, the high reactivity of the bare surface leads to much stronger adsorptions (with $E_{\mathrm{ads}}$ of -3.04 and $-8.05 \mathrm{eV}$, respectively) and to significantly distorted molecular structures (Figure S5). This suggests an activation of the molecule that would be sensitive to suffer bond dissociation.

Let us now analyze the possibility of electronic density transfer between the surface and each of the adsorbed molecules. The outcome of a Bader charge analysis ${ }^{52}$ shows an increase of the charge density on the surface, regardless of which AA was adsorbed and of its orientation relative to the surface. However, in the parallel adsorption, the magnitude of this change is small, amounting to less than $0.1 e$, and in some cases could even be assigned to uncertainties of numerical integration in the Bader atomic basins. In contrast, the surface Bader charge differences are expectedly higher when the adsorption occurs via $\mathrm{N}$-Ti bonding, and no longer negligible. In fact, the $\mathrm{N}$-bonded adsorption charge variations are around an order of magnitude higher than the ones related to parallel adsorption, supporting the conclusion that a chemical interaction between $\mathrm{N}$ and Ti. To test this hypothesis, we calculated the $\mathrm{N}-\mathrm{Ti}$ bond order for each adsorbed AA (see Table 1) and found values around 0.15, 
representative of weak bonding, and displaying very good correlation $\left(R^{2}=0.9975\right)$ with the $\mathrm{N}-\mathrm{Ti}$ distances. No correlation is found between the adsorption energies and the charge transferred from the adsorbate to the surface. The calculated projected density of states on the surface and AA bands are provided in Figures S7 and S8 for parallel and N-bonded adsorptions, respectively. In the case of the bare surface, the calculated band gap of $0.214 \mathrm{eV}$ compares well with the literature value ${ }^{60}, 0.24 \mathrm{eV}$, that was obtained with the PBE functional. Comparing the two adsorption modes, it is seen that many AA bands in the $\mathrm{N}$-adsorbed cases are in general shifted to more negative energy values than for the parallel adsorption, which is a consequence of the more favorable adsorption in the former.

Finally, we built 3D representations of the charge density differences. These provide a qualitative measure of the change in charge density on each point of real space in the region where the adsorption takes place, relatively to the surface and AA isolated from each other. The result is shown in Fig. 6 and reveals that some features are shared by all the AAs. The hydrogen atom of the amine group which is directed at the surface has lower density than in the molecule in gas form (denoted by the cyan color), in both adsorption configurations. In turn, the oxygen atoms of the surface closest to that hydrogen atom have their charge density increased (represented in gold color). On the parallel adsorption, we see the opposite effect in the carboxylic group of the AAs: the charge density of the oxygen atoms of this group increases, while the nearest surface oxygen atoms lose charge density. On the $\mathrm{N}$-bonded adsorption, the accumulation of shared electrons between $\mathrm{N}$ and $\mathrm{Ti}$, observed from the large blobs around the $\mathrm{N}-\mathrm{Ti}$ region, again indicates that a chemical bond is formed. Combining the information in the charge density profiles with the Bader charge calculations, one can infer that, upon parallel adsorption, the differences of charge density are the result of subtle electronic rearrangement on the surface and on the AA, which can be interpreted as a polarization of the MXene surface by the AA. The general features of our findings are thus in consonance with the ones found previously ${ }^{28}$ for the adsorption of $A A$ on graphene and hexagonal boron nitride. In particular, both studies concluded that when the AAs orient themselves parallel to the surface, $(i)$ the surfaces present little to no deformation, (ii) there is no charge transfer between the surface of the adsorbents and the AAs, and (iii) the adsorption is stronger for larger amino acids. Interestingly, for parallel adsorption, although the atom nearest to the surface is about $1 \AA$ closer in our case than in the case of graphene or BN, the adsorption energies on $\mathrm{BN}$ are an order of magnitude higher than on $\mathrm{Ti}_{2} \mathrm{CO}_{2}$, reflecting a higher affinity of $\mathrm{BN}$ towards amino acids. In fact, on $\mathrm{Ti}_{2} \mathrm{CO}_{2}$, the atoms nearest to the surface are hydrogens, rather than the hetero atoms, justifying the difference in the distances mentioned. 

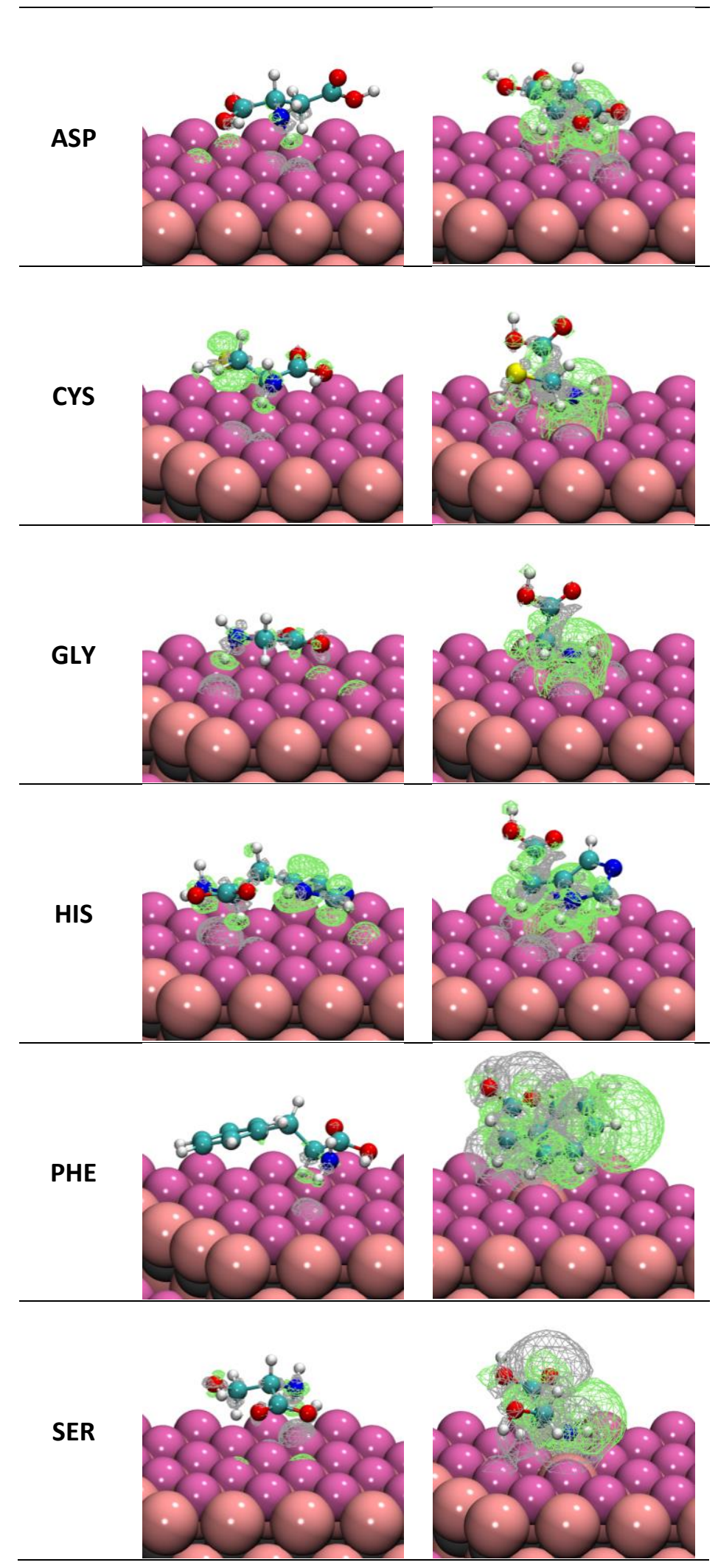

Figure 6 - Isosurfaces $\left(0.005 e / \AA^{3}\right)$ of charge density differences for the AAs@MXene systems compared to the isolated surface and amino acids, in the parallel (left) and $\mathrm{N}$-bonded (right) 
adsorption configurations. Color code for spheres as in Figure 3. The grey (green) wired region represents an increase (decrease) in charge density.

\section{Conclusions}

Using first-principles calculations based in the DFT framework, we have predicted the adsorption behavior of amino acids on the MXene $\mathrm{Ti}_{2} \mathrm{CO}_{2}$. At $T=0 \mathrm{~K}$, we found that most of the studied molecules prefer to adsorb via their amine $\mathrm{N}$ atom, chemically bound to a surface $\mathrm{Ti}$ atom, the latter being pulled towards the AA, sitting at a Ti-N distance of about $2.35 \AA$. The remaining molecules adsorb parallel to the surface, with the nearest atom and the alpha carbon lying around $2 \AA$ and $3 \AA$ away from the plane of oxygen atoms on the surface, respectively, while the adsorbent suffers practically no structural deformation. In both cases, the side chains of the AAs attempt to align with the plane of the surface. The absolute values of the calculated adsorption energies are between 0.5 and $1 \mathrm{eV}$ to show a linear correlation with the van der Waals volumes of the AAs. Extrapolating this correlation, one can infer that no amino acid should have an adsorption energy weaker than $-0.5 \mathrm{eV}$, and the AA which adsorbs more strongly on $\mathrm{Ti}_{2} \mathrm{CO}_{2}$ should be the one with the highest vdW volume, tryptophan, with an adsorption energy around $-1.1 \mathrm{eV}$. At ambient temperature and pressure, the preference for parallel or $\mathrm{N}$-bonded adsorption is reversed on most AAs, so that only ASP prefers to adsorb via $\mathrm{N}$-bonding, albeit less than $0.1 \mathrm{eV}$ more favorably than the parallel adsorption. This is also a clear indication of the very weak character of the Ti-N bond observed in this adsorption mode.

No significant charge transfer was found between the amino acids adsorbed with their planes parallel to the surface. However, the presence of the molecule does polarize the MXene in the nearby region: the charge density of the surface increases near the amine group and decreases near the carboxyl group of each amino acid. In contrast, $\mathrm{N}$-bonded adsorption leads to a small but noticeable electron transfer from the AA to the surface, of at most $0.2 e$, and to an accumulation of shared electrons between $\mathrm{N}$ and $\mathrm{Ti}$, indicating a chemical interaction between $\mathrm{Ti}$ and $\mathrm{N}$. The moderate adsorption energy values found, along with the lack of deformation of the amino acids and the surface, emphasize the capability of $\mathrm{Ti}_{2} \mathrm{CO}_{2}$ as an easily reusable biosensor for these molecules.

\section{Author Information}

\section{Corresponding Author}

José R. B. Gomes - CICECO - Aveiro Institute of Materials, Department of Chemistry, University of Aveiro, Campus Universitário de Santiago, 3810-193 Aveiro, Portugal; orcid.org/0000-00015993-1385; Email: jrgomes@ua.pt

\section{Authors:}

José D. Gouveia - CICECO - Aveiro Institute of Materials, Department of Chemistry, University of Aveiro, Campus Universitário de Santiago, 3810-193 Aveiro, Portugal; orcid.org/0000-00025099-7772

Gerard Novell-Leruth - CICECO - Aveiro Institute of Materials, Department of Chemistry, University of Aveiro, Campus Universitário de Santiago, 3810-193 Aveiro, Portugal; orcid.org/0000-0002-9018-0011 
Pedro M. L. S. Reis - CICECO - Aveiro Institute of Materials, Department of Chemistry, University of Aveiro, Campus Universitário de Santiago, 3810-193 Aveiro, Portugal; orcid.org/0000-0001-6380-2279/

Francesc Viñes - Departament de Ciència de Materials i Química Física \& Institut de Química Teòrica i Computacional (IQTCUB), Universitat de Barcelona, c/ Martí i Franqués 1, 08028 Barcelona, Spain; orcid.org/0000-0001-9987-8654

Francesc Illas - Departament de Ciència de Materials i Química Física \& Institut de Química Teòrica i Computacional (IQTCUB), Universitat de Barcelona, c/ Martí i Franqués 1, 08028 Barcelona, Spain; orcid.org/0000-0003-2104-6123

\section{Notes}

${ }^{a}$ These authors contributed equally.

The authors declare no competing financial interest.

\section{Associated Content}

The Supporting Information is available free of charge on the ACS Publications website at DOI:

Figure $\mathrm{S} 1$ with initial orientations of the amino acids on the $\mathrm{Ti}_{2} \mathrm{CO}_{2}$ surface. Figures $\mathrm{S} 2$ and $\mathrm{S} 5$ with the adsorbed configurations of $\mathrm{Gly}$ on $\mathrm{Ti}_{2} \mathrm{CO}_{2}$ and of Gly and $\mathrm{Phe}$ on $\mathrm{Ti}_{2} \mathrm{C}$, respectively. Figure S3 with details of the phase diagrams for the amino acids adsorbed parallel to the $\mathrm{Ti}_{2} \mathrm{CO}_{2}$ surface as a function of the AA partial pressures and temperature. Figures $\mathrm{S} 4$ and $\mathrm{S} 6$ with calculated infrared spectra of selected configurations of amino acids on the $\mathrm{Ti}_{2} \mathrm{CO}_{2}$ surface. Figures S7 and S8 with calculated projected density of states of selected configurations of amino acids on the $\mathrm{Ti}_{2} \mathrm{CO}_{2}$ surface. Tables $\mathrm{S} 1-\mathrm{S} 3$ with calculated wavenumbers for the amino acids adsorbed on the $\mathrm{Ti}_{2} \mathrm{CO}_{2}$ surface. Zip file with optimized coordinates.

\section{Acknowledgements}

This work was developed within the scope of the project CICECO-Aveiro Institute of Materials, Refs. UIDB/50011/2020 and UIDP/50011/2020, financed by national funds through the Fundação para a Ciência e a Tecnologia (FCT/MCTES) and co-financed by FEDER under the PT2020 Partnership Agreement. DG is thankful to project SILVIA with reference CENTRO-010145-FEDER-31002. The research carried out at the Universitat de Barcelona was supported by the Spanish Ministerio de Ciencia, Innovacion y Universidades (MICIUN) through the Excellence María de Maeztu MDM-2017-0767 and RTI2018-095460-B-I00 grants which involve FEDER funds. Partial support from Generalitat de Catalunya (2017SGR13 grant) is also acknowledged. FV thanks the Spanish MINECO for postdoctoral contracts under the Ramón y Cajal Program (RYC-2012-10129). F.I. acknowledges additional support from the 2015 ICREA Academia Award for Excellence in University Research. 


\section{References}

(1) Khan, K.; Tareen, A. K.; Aslam, M.; Wang, R.; Zhang, Y.; Mahmood, A.; Ouyang, Z.; Zhang, H.; Guo, Z. Recent developments in emerging two-dimensional materials and their applications. J. Mater. Chem. C 2020, 8 (2), 387-440 DOI: 10.1039/C9TC04187G.

(2) Naguib, M.; Kurtoglu, M.; Presser, V.; Lu, J.; Niu, J.; Heon, M.; Hultman, L.; Gogotsi, Y.; Barsoum, M. W. Two-dimensional nanocrystals produced by exfoliation of Ti3AIC2. Adv. Mater. 2011, 23 (37), 4248-4253 DOI: 10.1002/adma.201102306.

(3) Peng, J.; Chen, X.; Ong, W. J.; Zhao, X.; Li, N. Surface and Heterointerface Engineering of 2D MXenes and Their Nanocomposites: Insights into Electro- and Photocatalysis. Chem 2019, 5 (1), 18-50 DOI: 10.1016/j.chempr.2018.08.037.

(4) Anasori, B.; Lukatskaya, M. R.; Gogotsi, Y. 2D metal carbides and nitrides (MXenes) for energy storage. Nat. Rev. Mater. 2017, 2 (2), 16098 DOI: 10.1038/natrevmats.2016.98.

(5) Wang, H.; Wu, Y.; Yuan, X.; Zeng, G.; Zhou, J.; Wang, X.; Chew, J. W. Clay-Inspired MXene-Based Electrochemical Devices and Photo-Electrocatalyst: State-of-the-Art Progresses and Challenges. Adv. Mater. 2018, 30 (12), 1704561 DOI: 10.1002/adma.201704561.

(6) Jiang, Q.; Kurra, N.; Alhabeb, M.; Gogotsi, Y.; Alshareef, H. N. All Pseudocapacitive MXene-RuO 2 Asymmetric Supercapacitors. Adv. Energy Mater. 2018, 8 (13), 1703043 DOI: 10.1002/aenm.201703043.

(7) Naguib, M.; Come, J.; Dyatkin, B.; Presser, V.; Taberna, P.-L.; Simon, P.; Barsoum, M. W.; Gogotsi, Y. MXene: a promising transition metal carbide anode for lithium-ion batteries. Electrochem. commun. 2012, 16 (1), 61-64 DOI: 10.1016/j.elecom.2012.01.002.

(8) Yu, X.; Li, Y.; Cheng, J.; Liu, Z.; Li, Q.; Li, W.; Yang, X.; Xiao, B. Monolayer $\mathrm{Ti}_{2} \mathrm{CO}_{2}$ : A Promising Candidate for $\mathrm{NH}_{3}$ Sensor or Capturer with High Sensitivity and Selectivity. ACS Appl. Mater. Interfaces 2015, 7 (24), 13707-13713 DOI: 10.1021/acsami.5b03737.

(9) Morales-García, Á.; Fernández-Fernández, A.; Viñes, F.; Illas, F. $\mathrm{CO}_{2}$ abatement using two-dimensional MXene carbides. J. Mater. Chem. A 2018, 6 (8), 3381-3385 DOI: 10.1039/C7TA11379J.

(10) Morales-Salvador, R.; Morales-García, Á.; Viñes, F.; Illas, F. Correction: Two-dimensional nitrides as highly efficient potential candidates for $\mathrm{CO} 2$ capture and activation. Phys. Chem. Chem. Phys. 2018, 20 (37), 24490-24493 DOI: 10.1039/C8CP91852J.

(11) Persson, I.; Halim, J.; Lind, H.; Hansen, T. W.; Wagner, J. B.; Näslund, L.-Å.; Darakchieva, V.; Palisaitis, J.; Rosen, J.; Persson, P. O. Å. 2D Transition Metal Carbides (MXenes) for Carbon Capture. Adv. Mater. 2019, 31 (2), 1805472 DOI: 10.1002/adma.201805472.

(12) Zhao, L.; Dong, B.; Li, S.; Zhou, L.; Lai, L.; Wang, Z.; Zhao, S.; Han, M.; Gao, K.; Lu, M.; Xie, X; Chen, B.; Liu, Z.; Wang, X.; Zhang, H.; Li, H.; Liu, J.; Zhang, H.; Huang, X.; Huang, W. Interdiffusion Reaction-Assisted Hybridization of Two-Dimensional Metal-Organic Frameworks and $\mathrm{Ti}_{3} \mathrm{C}_{2} \mathrm{~T}_{x}$ Nanosheets for Electrocatalytic Oxygen Evolution. ACS Nano 2017, 11 (6), 5800-5807 DOI: 10.1021/acsnano.7b01409.

(13) Lu, C.; Tranca, D.; Zhang, J.; Rodríguez Hernández, F.; Su, Y.; Zhuang, X.; Zhang, F.; Seifert, G.; Feng, X. Molybdenum Carbide-Embedded Nitrogen-Doped Porous Carbon Nanosheets as Electrocatalysts for Water Splitting in Alkaline Media. ACS Nano 2017, 11 (4), 3933-3942 DOI: 10.1021/acsnano.7b00365. 
(14) Gao, G.; O'Mullane, A. P.; Du, A. 2D MXenes: A New Family of Promising Catalysts for the Hydrogen Evolution Reaction. ACS Catal. 2017, 7 (1), 494-500 DOI:

10.1021/acscatal.6b02754.

(15) Ling, C.; Shi, L.; Ouyang, Y.; Chen, Q.; Wang, J. Transition Metal-Promoted $\mathrm{V}_{2} \mathrm{CO}_{2}$ (MXenes): A New and Highly Active Catalyst for Hydrogen Evolution Reaction. Adv. Sci. 2016 DOI: 10.1002/advs.201600180.

(16) Ma, L.; Ting, L. R. L.; Molinari, V.; Giordano, C.; Yeo, B. S. Efficient hydrogen evolution reaction catalyzed by molybdenum carbide and molybdenum nitride nanocatalysts synthesized via the urea glass route. J. Mater. Chem. A 2015, 3 (16), 8361-8368 DOI: 10.1039/C5TA00139K.

(17) Azofra, L. M.; Li, N.; MacFarlane, D. R.; Sun, C. Promising prospects for 2D $d^{2}-d^{4} M_{3} C_{2}$ transition metal carbides (MXenes) in $\mathrm{N}_{2}$ capture and conversion into ammonia. Energy Environ. Sci. 2016, 9 (8), 2545-2549 DOI: 10.1039/C6EE01800A.

(18) Deeva, E. B.; Kurlov, A.; Abdala, P. M.; Lebedev, D.; Kim, S. M.; Gordon, C. P.; Tsoukalou, A.; Fedorov, A.; Müller, C. R. In Situ XANES/XRD Study of the Structural Stability of TwoDimensional Molybdenum Carbide $\mathrm{Mo}_{2} \mathrm{CT}_{\mathrm{x}}$ : Implications for the Catalytic Activity in the Water-Gas Shift Reaction. Chem. Mater. 2019, 31 (12), 4505-4513 DOI: 10.1021/acs.chemmater.9b01105.

(19) Gouveia, J. D.; Morales-García, Á.; Viñes, F.; Illas, F.; Gomes, J. R. B. MXenes as promising catalysts for water dissociation. Appl. Catal. B Environ. 2020, 260, 118191 DOI: 10.1016/j.apcatb.2019.118191.

(20) Gouveia, J. D.; Morales-García, Á.; Viñes, F.; Gomes, J. R. B.; Illas, F. Facile Heterogeneously Catalyzed Nitrogen Fixation by MXenes. ACS Catal. 2020, 10 (9), 5049-5056 DOI: 10.1021/acscatal.0c00935.

(21) Hart, J. L.; Hantanasirisakul, K.; Lang, A. C.; Anasori, B.; Pinto, D.; Pivak, Y.; van Omme, J. T.; May, S. J.; Gogotsi, Y.; Taheri, M. L. Control of MXenes' electronic properties through termination and intercalation. Nat. Commun. 2019, 10 (1), 522 DOI: 10.1038/s41467018-08169-8.

(22) Liu, W.; Xu, S.; Hu, B.; Wang, X. A novel potential modulated amino acid sensing chip modified by MXene for total internal reflection imaging ellipsometry biosensor. Proc. IEEE Int. Conf. Micro Electro Mech. Syst. 2018, 2018-Janua (January), 348-351 DOI: 10.1109/MEMSYS.2018.8346558.

(23) Rozmysłowska-Wojciechowska, A.; Wojciechowski, T.; Ziemkowska, W.; Chlubny, L.; Olszyna, A.; Jastrzębska, A. M. Surface interactions between $2 \mathrm{D} \mathrm{Ti}{ }_{3} \mathrm{C}_{2} / \mathrm{Ti}_{2} \mathrm{C}$ MXenes and lysozyme. Appl. Surf. Sci. 2019, 473, 409-418 DOI: 10.1016/j.apsusc.2018.12.081.

(24) Mojtabavi, M.; VahidMohammadi, A.; Liang, W.; Beidaghi, M.; Wanunu, M. SingleMolecule Sensing Using Nanopores in Two-Dimensional Transition Metal Carbide (MXene) Membranes. ACS Nano 2019, 13 (3), 3042-3053 DOI: 10.1021/acsnano.8b08017.

(25) Long, M.; Rack, H. . Titanium alloys in total joint replacement-a materials science perspective. Biomaterials 1998, 19 (18), 1621-1639 DOI: 10.1016/S01429612(97)00146-4.

(26) Vitale, F.; Driscoll, N.; Murphy, B. Biomedical Applications of MXenes. In 2D Metal Carbides and Nitrides (MXenes): Structure, Properties and Applications; Anasori, B., 
Gogotsi, Y., Eds.; Springer International Publishing: Cham, 2019; pp 503-524.

(27) Chen, C.; Boota, M.; Urbankowski, P.; Anasori, B.; Miao, L.; Jiang, J.; Gogotsi, Y. Effect of glycine functionalization of 2D titanium carbide (MXene) on charge storage. J. Mater. Chem. A 2018, 6 (11), 4617-4622 DOI: 10.1039/C7TA11347A.

(28) Singla, P.; Riyaz, M.; Singhal, S.; Goel, N. Theoretical study of adsorption of amino acids on graphene and $\mathrm{BN}$ sheet in gas and aqueous phase with empirical DFT dispersion correction. Phys. Chem. Chem. Phys. 2016, 18 (7), 5597-5604 DOI: 10.1039/c5cp07078c.

(29) Kresse, G.; Hafner, J. Ab initio molecular dynamics for liquid metals. Phys. Rev. B 1993, 47 (1), 558-561 DOI: 10.1103/PhysRevB.47.558.

(30) Kresse, G.; Hafner, J. Ab initio molecular-dynamics simulation of the liquid-metalamorphous-semiconductor transition in germanium. Phys. Rev. B 1994, 49 (20), 1425114269 DOI: 10.1103/PhysRevB.49.14251.

(31) Kresse, G.; Furthmüller, J. Efficient iterative schemes for ab initio total-energy calculations using a plane-wave basis set. Phys. Rev. B 1996, 54 (16), 11169-11186 DOI: 10.1103/PhysRevB.54.11169.

(32) Kresse, G.; Furthmüller, J. Efficiency of ab-initio total energy calculations for metals and semiconductors using a plane-wave basis set. Comput. Mater. Sci. 1996, 6 (1), 15-50 DOI: 10.1016/0927-0256(96)00008-0.

(33) Perdew, J. P.; Burke, K.; Ernzerhof, M. Generalized Gradient Approximation Made Simple. Phys. Rev. Lett. 1996, 77 (18), 3865-3868 DOI: 10.1103/PhysRevLett.77.3865.

(34) Grimme, S.; Antony, J.; Ehrlich, S.; Krieg, H. A consistent and accurate ab initio parametrization of density functional dispersion correction (DFT-D) for the 94 elements H-Pu. J. Chem. Phys. 2010, 132 (15), 154104 DOI: 10.1063/1.3382344.

(35) Grimme, S.; Ehrlich, S.; Goerigk, L. Effect of the damping function in dispersion corrected density functional theory. J. Comput. Chem. 2011, 32 (7), 1456-1465 DOI: $10.1002 / j c c .21759$.

(36) Fajín, J. L. C.; Gomes, J. R. B.; Cordeiro, M. N. D. S. DFT Study of the Adsorption of d-(I)Cysteine on Flat and Chiral Stepped Gold Surfaces. Langmuir 2013, 29 (28), 8856-8864 DOI: 10.1021/la401057f.

(37) Ramalho, J. P. P.; Gomes, J. R. B.; Illas, F. Accounting for van der Waals interactions between adsorbates and surfaces in density functional theory based calculations: selected examples. RSC Adv. 2013, 3 (32), 13085 DOI: 10.1039/c3ra40713f.

(38) Viñes, F.; Lamiel-García, O. Approaching the Quantitative Description of Enantioselective Adsorption by the Density Functional Theory Means. J. Phys. Chem. $C$ 2019, 123 (18), 11714-11722 DOI: 10.1021/acs.jpcc.9b01463.

(39) Neugebauer, J.; Scheffler, M. Adsorbate-substrate and adsorbate-adsorbate interactions of $\mathrm{Na}$ and $\mathrm{K}$ adlayers on Al(111). Phys. Rev. B 1992, 46 (24), 16067-16080 DOI: 10.1103/PhysRevB.46.16067.

(40) Blöchl, P. E. Projector augmented-wave method. Phys. Rev. B 1994, 50 (24), 1795317979 DOI: 10.1103/PhysRevB.50.17953.

(41) Monkhorst, H. J.; Pack, J. D. Special points for Brillouin-zone integrations. Phys. Rev. B 1976, 13 (12), 5188-5192 DOI: 10.1103/PhysRevB.13.5188. 
(42) Zhang, N.; Hong, Y.; Yazdanparast, S.; Asle Zaeem, M. Superior structural, elastic and electronic properties of 2D titanium nitride MXenes over carbide MXenes: a comprehensive first principles study. 2D Mater. 2018, 5 (4), 045004 DOI: 10.1088/20531583/aacfb3.

(43) Grimme, S.; Bannwarth, C.; Shushkov, P. A Robust and Accurate Tight-Binding Quantum Chemical Method for Structures, Vibrational Frequencies, and Noncovalent Interactions of Large Molecular Systems Parametrized for All spd-Block Elements $(Z=1-86)$. J. Chem. Theory Comput. 2017, 13 (5), 1989-2009 DOI: 10.1021/acs.jctc.7b00118.

(44) Bannwarth, C.; Ehlert, S.; Grimme, S. GFN2-xTB-An Accurate and Broadly Parametrized Self-Consistent Tight-Binding Quantum Chemical Method with Multipole Electrostatics and Density-Dependent Dispersion Contributions. J. Chem. Theory Comput. 2019, 15 (3), 1652-1671 DOI: 10.1021/acs.jctc.8b01176.

(45) Laidler, K. J. Chemical Kinetics, 3rd ed.; Harper Collins: New York, 1987.

(46) McQuarrie, D. A. Statistical Mechanics; Harper \& Row: New York, 1975.

(47) Herzberg, G. Molecular spectra and molecular structure. Vol.2: Infrared and Raman spectra of polyatomic molecules; Van Nostrand: New York, 1945.

(48) Atkins, P. W.; De Paula, J. Atkins' Physical Chemistry; Oxford Univ. Press: Oxford, 2006.

(49) Lourenço, M. A. O.; Ferreira, P.; Gomes, J. R. B. Flue gas adsorption on periodic mesoporous phenylene-silica: a DFT approach. Phys. Chem. Chem. Phys. 2018, 20 (24), 16686-16694 DOI: 10.1039/C8CP02589D.

(50) Kunkel, C.; Viñes, F.; Illas, F. Transition metal carbides as novel materials for $\mathrm{CO} 2$ capture, storage, and activation. Energy Environ. Sci. 2016, 9 (1), 141-144 DOI: 10.1039/C5EE03649F.

(51) Kunkel, C.; Viñes, F.; Lourenço, M. A. O.; Ferreira, P.; Gomes, J. R. B.; Illas, F. Selectivity for $\mathrm{CO}_{2}$ over $\mathrm{CH}_{4}$ on a functionalized periodic mesoporous phenylene-silica explained by transition state theory. Chem. Phys. Lett. 2017, 671, 161-164 DOI: 10.1016/j.cplett.2017.01.033.

(52) Henkelman, G.; Arnaldsson, A.; Jónsson, H. A fast and robust algorithm for Bader decomposition of charge density. Comput. Mater. Sci. 2006, 36 (3), 354-360 DOI: 10.1016/j.commatsci.2005.04.010.

(53) Schulte, F. K. Chemical potentials and workfunctions of metal surfaces in the theory of an inhomogeneous electron gas. J. Phys. C Solid State Phys. 1974, 7 (20), L370-L373 DOI: 10.1088/0022-3719/7/20/003.

(54) Kasalová, V.; Allen, W. D.; Schaefer, H. F.; Czinki, E.; Császár, A. G. Molecular structures of the two most stable conformers of free glycine. J. Comput. Chem. 2007, 28 (8), 13731383 DOI: $10.1002 /$ jcc.20680.

(55) Morales-García, Á.; Mayans-Llorach, M.; Viñes, F.; Illas, F. Thickness biased capture of $\mathrm{CO}_{2}$ on carbide MXenes. Phys. Chem. Chem. Phys. 2019, 21 (41), 23136-23142 DOI: 10.1039/С9CP04833B.

(56) Stepanian, S. G.; Reva, I. D.; Radchenko, E. D.; Rosado, M. T. S.; Duarte, M. L. T. S.; Fausto, R.; Adamowicz, L. Matrix-Isolation Infrared and Theoretical Studies of the Glycine Conformers. J. Phys. Chem. A 1998, 102 (6), 1041-1054 DOI: 10.1021/jp973397a. 
(57) Carrasco, J.; Michaelides, A.; Forster, M.; Haq, S.; Raval, R.; Hodgson, A. A onedimensional ice structure built from pentagons. Nat. Mater. 2009, 8 (5), 427-431 DOI: 10.1038/nmat2403.

(58) Darby, N. J.; Creighton, T. E. Protein structure; IRL Press at Oxford University Press: Oxford, 1993.

(59) Manz, T. A. Introducing DDEC6 atomic population analysis: Part 3. Comprehensive method to compute bond orders. RSC Adv. 2017 DOI: 10.1039/c7ra07400j.

(60) Xie, Y.; Kent, P. R. C. Hybrid density functional study of structural and electronic properties of functionalized $\mathrm{Ti}_{n+1} \mathrm{X}_{\mathrm{n}}(\mathrm{X}=\mathrm{C}, \mathrm{N})$ monolayers. Phys. Rev. B 2013, 87 (23), 235441 DOI: 10.1103/PhysRevB.87.235441. 
For Table of Contents Only
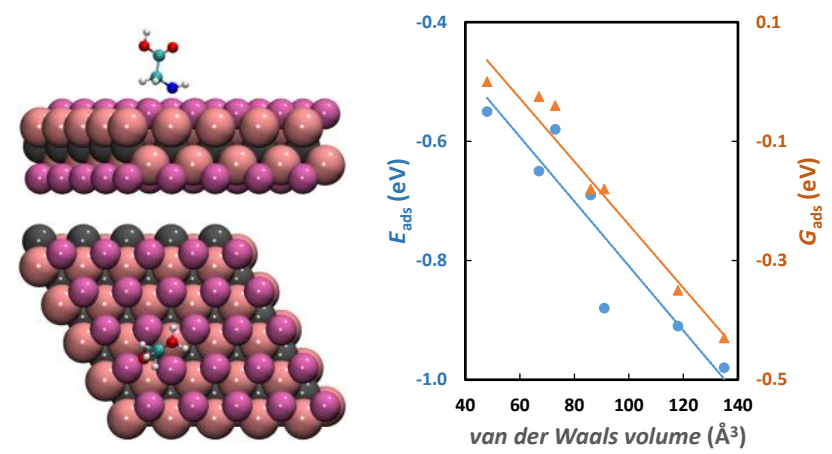